\title{
Marked enhancement of Acinetobacter sp. organophosphorus hydrolase activity by a single residue substitution Ile211 Ala
}

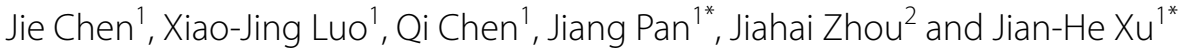

\begin{abstract}
Background: The activity of organophosphorus hydrolase (OPH) that catalyzes the hydrolysis of neurotoxic organophosphates (OPs) was reported to evolve from lactonase.

Results: In this study, a putative OPH from Acinetobacter sp. (AbOPH) exhibited high lactonase activity with latent $\mathrm{OPH}$ activity. Sequence alignment and phylogenetic tree analysis revealed the unique status of $\mathrm{AbOPH}$ in evolution. The crystal structure of $\mathrm{AbOPH}$ was determined at $2.0 \AA$ resolution and a semi-rational design was performed to enhance the $\mathrm{OPH}$ activity of $\mathrm{AbOPH}$ through a consensus sequence approach. Compared with wild-type $\mathrm{AbOPH}$, which exhibited undetectable activity toward methyl-parathion (MP), the best variant $A b O \mathrm{PH}_{1211 \mathrm{~A}}$ showed markedly improved catalytic efficiency $\left(1.1 \mu \mathrm{mol} \mathrm{min}{ }^{-1} \mathrm{mg}_{\text {protein }}^{-1}\right)$ toward MP. Docking studies suggested that the mutation lle211Ala affects substrate recognition and stabilizes substrate conformation.
\end{abstract}

Conclusions: This result presents the emergence of new enzyme function by a simple mutation strategy and confirms the high possibility that OPH was evolved from its lactonase ancestor.

Keywords: Crystal structure, Organophosphorus hydrolase, Lactonase, Semi-rational design, Site-directed mutagenesis

\section{Background}

Organophosphates (OPs) are common neurotoxic compounds (Singh 2008) that have been extensively used as agricultural insecticides (Raushel 2002). Massive use of these pesticides has brought serious threats to environmental safety and human health (Sapozhnikova et al. 2004, 2005). Enzymatic degradation of OPs was considered as a desirable decontamination method with many advantages, such as being economic, efficient and environmentally friendly (Singh and Walker 2006). A number of enzymes capable of degrading OPs have been discovered during the past years, including organophosphorus acid anhydrolases (OPAAs; EC 3.4.13.9) (Cheng et al. 1999; Vyas et al. 2010), phosphotriesterases (PTEs; EC

\footnotetext{
*Correspondence: panjiang@ecust.edu.cn; jianhexu@ecust.edu.cn 1 State Key Laboratory of Bioreactor Engineering, Shanghai Collaborative Innovation Center for Biomanufacturing, East China University of Science and Technology, Shanghai 200237, China

Full list of author information is available at the end of the article
}

3.1.8.1) (Omburo et al. 1992), paraoxonases (PONs; EC 3.1.8.1) (Ben-David et al. 2012), phosphotriesterase-like lactonases (PLLs; EC 3.1.1.81) (Afriat et al. 2006), and methyl-parathion hydrolases (MPHs; EC 3.1.8.1) (Hong et al. 2005).

In recent years, clarifying the evolutionary pathway (Bar-Rogovsky et al. 2013; Luo et al. 2014) and structure-activity relationship (Dong et al. 2005; Gotthard et al. 2013) of organophosphorus hydrolase (OPH) has been extensively studied. Phosphotriesterase (PTE) from Brevundimonas diminuta was the best-characterized and potentially important $\mathrm{OPH}$, which exhibited near diffusion-limit reaction rate $\left(10^{7}-10^{8} \mathrm{M}^{-1} \mathrm{~s}^{-1}\right)$ to ethyl-paraoxon (Omburo et al. 1992). PTE was reported to emerge from native lactonases (PLLs) with promiscuous $\mathrm{OPH}$ activity (Merone et al. 2010; Elias et al. 2008; Hawwa et al. 2009). In addition, mammalian paraoxonase (PON) also showed native lactonase activity (Khersonsky and Tawfik 2005), which was believed to evolve from mammalian

\section{Springer}

(c) 2015 Chen et al. This article is distributed under the terms of the Creative Commons Attribution 4.0 International License (http://creativecommons.org/licenses/by/4.0/), which permits unrestricted use, distribution, and reproduction in any medium, provided you give appropriate credit to the original author(s) and the source, provide a link to the Creative Commons license, and indicate if changes were made. 
paraoxonase ancestor with bifunctionality (HSLase and $\delta$-/ $\gamma$-lactonase) (Bar-Rogovsky et al. 2013).

The MPH isolated from Pseudomonas sp. strain WBC-3 catalyzes the degradation of the organophosphate pesticide methyl-parathion (MP) with a high efficiency $\left(k_{\text {cat }} / K_{\mathrm{M}} \sim 10^{6} \mathrm{M}^{-1} \mathrm{~s}^{-1}\right)$ (Dong et al. 2005). The structure of $\mathrm{MPH}$ (PDB ID: 1P9E) has been resolved, revealing a typical $\alpha \beta / \beta \alpha$ sandwich fold structure of metallo- $\beta$ lactamase superfamily (Dong et al. 2005). In parallel with $\mathrm{MPH}$, a newly identified $\mathrm{OPH}, \mathrm{OPHC} 2$, isolated from Pseudomonas pseudoalcaligenes, is a thermostable $\mathrm{OPH}$ $\left(T_{\mathrm{m}}=97.8 \pm 3.2^{\circ} \mathrm{C}\right)$ (Gotthard et al. 2013) in $\beta$-lactamase superfamily, which shares $46 \%$ sequence identity with $\mathrm{MPH}$. PoOPH, a newly discovered lactonase from Pseudomonas oleovorans, exhibited high lactonase and esterase activities, but low OPH activity (Luo et al. 2014). PoOPH shares $98.5 \%$ sequence identity with OPHC2. By simple double mutations His250Ile/Ile263Trp, PoOPH was switched into an efficient $\mathrm{OPH}$, displaying 6962-fold improvement in catalytic efficiency toward MP (Luo et al. 2014). The study proves the emergence of efficient and robust enzymes for OP detoxification by OPH activity evolution in the $\beta$-lactamase superfamily.

In this study, a distinctive putative $\mathrm{OPH}(A b \mathrm{OPH})$ identified from Acinetobacter sp. exhibited high lactonase activity with latent $\mathrm{OPH}$ activity. Compared with OPHC2 and $\mathrm{MPH}$ in $\beta$-lactamase superfamily, $A b \mathrm{OPH}$ acts as a separate branch in the evolutionary tree and there are four additional amino acids (Gly206, Thr207, Val208 and Glu209) in its sequence. A semi-rational design was performed for $A b \mathrm{OPH}$ based on its crystal structure and sequence analysis to find the key residues that affect its $\mathrm{OPH}$ activity. The best variant $A b \mathrm{OPH}_{\mathrm{I} 211 \mathrm{~A}}$ was characterized and the possible mechanism for the improvement of $\mathrm{OPH}$ activity was investigated by molecular docking studies.

\section{Methods}

\section{Chemicals and strains}

OPs were purchased from Shanghai Pesticide Research Center. para-Nitrophenyl butyrate ( $p$ NPB) was synthesized in our laboratory. 3,4-Dihydrocoumarin, $\delta$-nonanolactone and $\gamma$-decanolactone were obtained from TCI Co., Ltd. (Tokyo, Japan). All chemicals used for crystallization were purchased from Sigma-Aldrich Co. (St. Louis, MO, USA). Other chemicals with reagent grade or better quality were obtained from commercial sources. Escherichia coli (E. coli) DH5 $\alpha$ and E. coli BL21(DE3) (Novagen, Germany) were used for gene amplification and expression, respectively (Du et al. 2014). Acinetobacter sp. (NBRC 100985) used in this study was purchased from NBRC (NITE Biological Resource Center).

\section{AbOPH cloning and site-directed mutagenesis}

$A b \mathrm{OPH}$ gene was amplified by PCR using whole genome of Acinetobacter sp. as template with the following primers: 5'-GGGTTTCATATGATGCTAAAAAATAGAC-3' (forward) and 5'-CCCAAGCTTATCTTTAAAATG GATCGGA-3' (reverse) (Luo et al. 2014). The PCR fragment was subsequently cloned into expression vector $\mathrm{pET}-28 \mathrm{a}(+)$ to generate the recombinant plasmid pET28a-AbOPH. Site-directed mutations of $A b \mathrm{OPH}$ were constructed using QuickChange ${ }^{\circledR}$ Site-Directed Mutagenesis Kit (Stratagene, USA), and the plasmids carrying the gene of $A b \mathrm{OPH}$ and the desired mutations were transformed into $E$. coli $\mathrm{DH} 5 \alpha$ for amplification and then transformed into E. coli BL21(DE3) cells for expression.

\section{Protein expression and purification}

A single colony of recombinant strain was cultivated in LB medium containing $50 \mu \mathrm{g} / \mathrm{mL}$ kanamycin at $37{ }^{\circ} \mathrm{C}$. When $\mathrm{OD}_{600}$ reached $0.6-0.8$, the cultivation temperature was decreased to $16{ }^{\circ} \mathrm{C}$ and isopropyl $\beta$-Dthiogalactopyranoside (IPTG) was added with a final concentration of $0.5 \mathrm{mM}$ to induce protein expression. After $20 \mathrm{~h}$, the cells from $1 \mathrm{~L}$ culture were collected by centrifugation $(8800 \times g)$ at $16{ }^{\circ} \mathrm{C}$, resuspended in buffer A $(20 \mathrm{mM}$ Tris- $\mathrm{HCl}, 500 \mathrm{mM} \mathrm{NaCl}, 20 \mathrm{mM}$ imidazole, $\mathrm{pH}$ 8.0) and lysed by French Press. The cell lysate was centrifuged at $30,000 \times g, 4{ }^{\circ} \mathrm{C}$ for $1 \mathrm{~h}$; then the supernatant was loaded onto a $\mathrm{Ni}^{2+}$-NTA affinity column $(5 \mathrm{~mL}$, GE Healthcare Co.). After prewashing with buffer A, the recombinant protein was eluted with buffer A containing imidazole with linear gradient from 10 to $500 \mathrm{mM}$. The target protein collected was then loaded onto a HiLoad 16/60 Superdex 75 preparative column $(120 \mathrm{~mL}$, GE Healthcare Co.) and eluted by Tris- $\mathrm{HCl}$ buffer $(25 \mathrm{mM}$, $\mathrm{pH}$ 7.5) containing $150 \mathrm{mM} \mathrm{NaCl}$ and $1 \mathrm{mM}$ dithiothreitol for protein condensation. SDS-PAGE analysis of the eluted protein revealed over $90 \%$ purity of the target protein. The final volume and concentration of the concentrated $A b \mathrm{OPH}$ protein were $1 \mathrm{~mL}$ and $14 \mathrm{mg} / \mathrm{mL}$, respectively.

\section{Crystallization, data collection and structure refinement}

Crystallization of $A b \mathrm{OPH}$ was carried out at $4{ }^{\circ} \mathrm{C}$ using sitting-drop vapor diffusion method with crystallization buffer containing $0.02 \mathrm{M}$ D-glucose, $0.02 \mathrm{M}$ D-mannose, $0.02 \mathrm{M}$ D-galactose, $0.02 \mathrm{M}$ L-fucose, $0.02 \mathrm{M} \mathrm{D}$-xylose, $0.02 \mathrm{M} \mathrm{N}$-acetyl-D-glucosamine, MES/Imidazole $(0.1 \mathrm{M}$, $\mathrm{pH}$ 6.5), $12.5 \%$ (w/v) PEG1000, $12.5 \%(\mathrm{w} / \mathrm{v})$ PEG3350 and $12.5 \%(v / v)$ 2-methyl-2,4-pentanediol. The protein concentration for crystallization was $14 \mathrm{mg} / \mathrm{mL}$. Crystals that appeared were transferred to cryoprotectant solution composed of mother liquors supplemented with $5 \%(v / v)$ ethylene glycol prior to flash cooling in liquid 
nitrogen. X-ray diffraction data were collected with flashfrozen crystals (at $100 \mathrm{~K}$ in a stream of nitrogen gas) on a RaxisIV++ imaging plate (Rigaku, TX, USA) using an in-house Rigaku MicroMax-007 HF rotating-anode X-ray generator operating at $40 \mathrm{kV}$ and $30 \mathrm{~mA}$. The intensity sets were indexed, integrated and scaled with the HKL2000 package (Otwinowski and Minor 1997). The initial structure of $A b \mathrm{OPH}$ was determined by molecular replacement with PHASER (McCoy et al. 2007) using OPHC2 (PDBID: 4LE6) as the template. Several cycles of refinement were carried out using Phenix and Coot (Murshudov et al. 1997; Emsley and Cowtan 2004).

\section{Activity assay and kinetic parameters determination}

The enzyme activities toward various OPs (methylparathion, ethyl-paraoxon, malathion, fenitrothion, dimethoate and diazinon), lactones (3,4-dihydrocoumarin, $\delta$-decanolactone and $\gamma$-nonanolactone) and esters (2-naphthyl acetate and para-nitrophenyl butyrate) were determined as described (Gotthard et al. 2013; Khersonsky and Tawfik 2005; Dumas et al. 1989; Zhang et al. 2012; Roodveldt and Tawfik 2005). The kinetic parameters were determined by measuring activities toward MP at concentrations from 0.01 to $1 \mathrm{mM}$ and fitting data to the Michaelis-Menten equation.

\section{Homology modeling and molecular docking}

The structure of $A b \mathrm{OPH}_{\mathrm{I} 211 \mathrm{~A}}$ was modeled using $A b \mathrm{OPH}$ crystal structure as the template on SWISS-MODEL sever (http://swissmodel.expasy.org/). The substrate MP was docked into $A b \mathrm{OPH}_{\mathrm{I} 211 \mathrm{~A}}$ and $A b \mathrm{OPH}$, respectively, using Discovery Studio 2.5 software. Two zinc ions were selected as binding site. The radius of the SBD site sphere was set as $9 \AA$. LibDock protocol was used in further docking simulation. The conformations from the calculation that conflict with the catalytic mechanism were initially ruled out. The best-scoring conformation was then subjected to detailed analysis.

\section{Results and discussion}

\section{Identification and characterization of a putative $\mathrm{OPH}$}

To identify new enzymes with potential OPH activities, OPHC2 was chosen as a probe to search for homolog proteins based on BLAST (Basic Local Alignment Search Tool) Web server in the NCBI (National Center for Biotechnology Information) database. Eleven OPHC2-like proteins with sequence identity ranging from 40 to $98 \%$ were selected as candidates (Fig. 1a, Additional file 1: Figure S1). All of these proteins belong to $\beta$-lactamase superfamily and show entirely conserved metal ion coordination residues. From the evolutionary tree analysis, two putative OPHs $(A b \mathrm{OPH}$ and $\mathrm{SmOPH})$ act as separate branches, belonging to neither $\mathrm{MPH}$ nor $\mathrm{OPHC} 2$ clade (Fig. 1b) (Luo et al. 2014). Based on the results of sequence alignment, the $A b \mathrm{OPH}$ seems quite special since there are four additional amino acids (Gly206, Thr207, Val208 and Glu209) in its sequence, which do not exist in other putative OPHs. The putative $\mathrm{OPH}$ from Acinetobacter sp., hereafter designated as $A b \mathrm{OPH}$, shares merely $44 \%$ sequence identity with OPHC2 and $38 \%$ with $\mathrm{MPH}$. The $A b \mathrm{OPH}$ was heterologously overexpressed in $E$. coli, purified and characterized. The molecular weight of purified $A b \mathrm{OPH}$ was about $37 \mathrm{kDa}$ according to SDS-PAGE analysis (Additional file 1: Figure S2A). Similar to PoOPH (Luo et al. 2014), $A b \mathrm{OPH}$ also exhibited some functional promiscuity, with high lactonase activity $\left(996 \mu \mathrm{mol} \mathrm{min}{ }^{-1} \mathrm{mg}^{-1}\right.$ toward 3,4-dihydrocoumarin) and latent $\mathrm{OPH}$ activity (only $0.04 \mu \mathrm{mol} \mathrm{min}^{-1} \mathrm{mg}^{-1}$ toward fenitrothion and no activity was detected toward MP).

\section{Crystal structure of $A b O P H$}

The crystal structure of $A b \mathrm{OPH}$ was solved at $2.0 \AA$ resolution (PDB ID: 4XUK) (Additional file 1: Figure S2B). The crystal contains two molecules in the asymmetric unit and belongs to the space group $P 2{ }_{1} 2_{1} 2_{1}$ with unit cell parameters $a=76.8, b=82.7, c=99.8$ and $\alpha=\beta=\gamma=90^{\circ}$. Refinement of the structure finally converged with an $R$-work of 0.204 and $R$-free of 0.238 . The final model comprised 291 amino acids, 2 zinc ions and 242 water molecules. Because the residues 1-47 were absent in the electron density maps, they were not modeled. The monomer of $A b \mathrm{OPH}$ is roughly ellipsoidal with approximate dimensions of $52 \AA \times 50 \AA \times 32 \AA$. Data collection and refinement statistics are summarized in Table 1.

$A b \mathrm{OPH}$ forms a homodimer in the asymmetric unit (Fig. 2a) and the structure could be described as $\alpha \beta / \beta \alpha$ sandwich topology (Fig. 2b), analogous to other members of $\beta$-lactamase superfamily (Luo et al. 2014; Gotthard et al. 2013). Each asymmetric unit is composed of a $\beta$-lactamase-like domain and the four additional residues 206-209 are located in helix $\alpha 5$. The binuclear metal center locates between the inner $\beta$-sheets of the $\alpha \beta / \beta \alpha$ sandwich. The two zinc ions are separated by a distance of $3.2 \AA$. The buried $\alpha$ zinc ion is coordinated by Asp159, His160, Asp265 and His312 and the less buried $\beta$ zinc ion is coordinated by His244, His155, His157, Asp265 and a water molecule. Both metals are bridged by a putative catalytic water molecule (Fig. 2c).

Structural comparison of $A b \mathrm{OPH}$ with other OPHs showed that the overall conformation and the active 


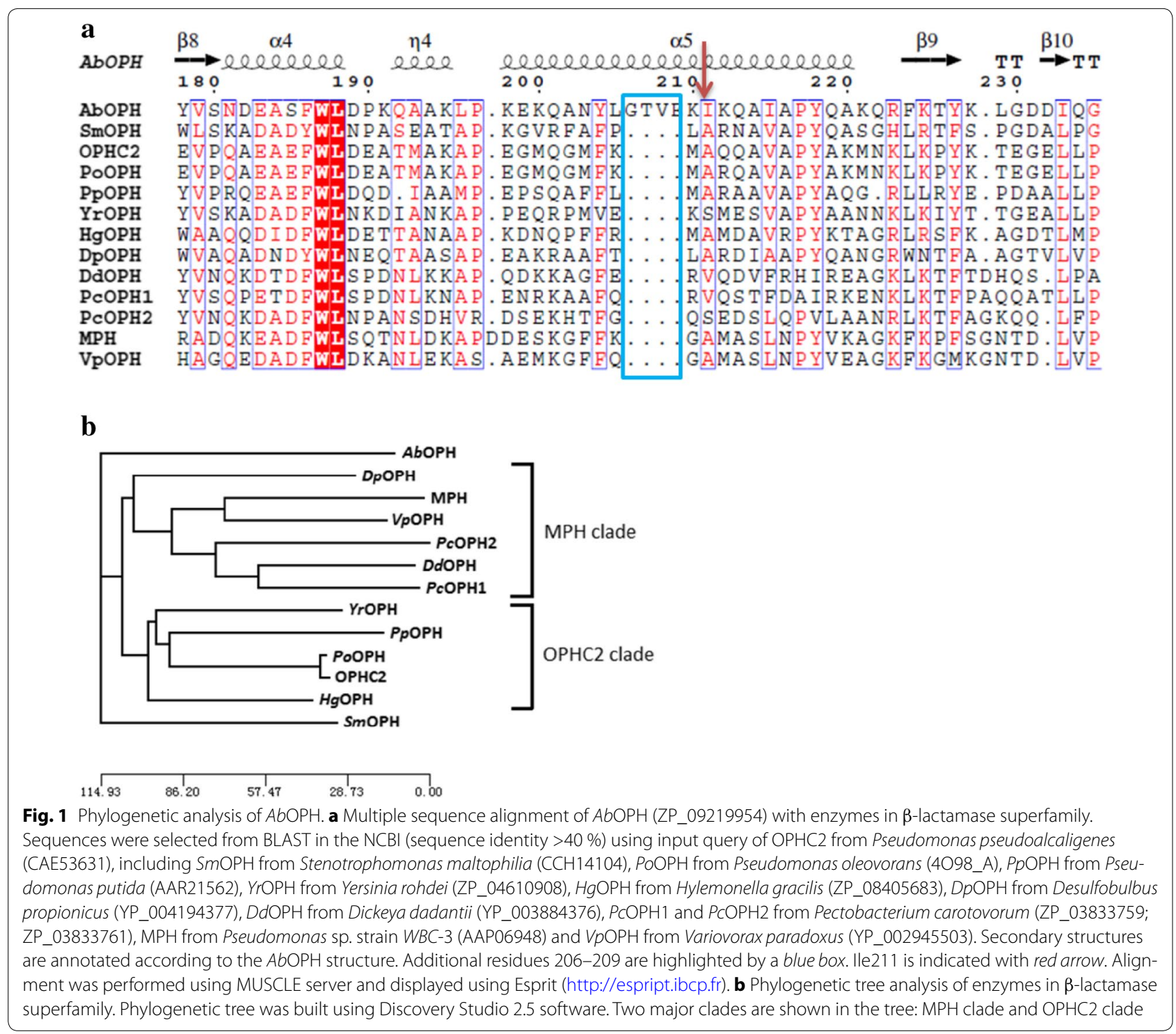

center of $A b \mathrm{OPH}$ were similar to $\mathrm{OPHC} 2$ and $\mathrm{MPH}$ (Additional file 1: Figure S3), while the $\mathrm{OPH}$ activity of $A b \mathrm{OPH}$ was considerably lower than that of OPHC2 and $\mathrm{MPH}$. Considering the high conformation similarities in contrast to huge differences of $\mathrm{OPH}$ activity between $A b \mathrm{OPH}, \mathrm{OPHC} 2$ and $\mathrm{MPH}$, a semi-rational design based on consensus sequence approach was performed to identify the key residues that depress the $\mathrm{OPH}$ activity of $A b \mathrm{OPH}$.

\section{Site-directed mutagenesis of $A b O P H$}

Nineteen conserved residues of $A b \mathrm{OPH}$ (Additional file 1: Figure S4) within $10 \AA$ from the active center were mutated into the corresponding amino acid residues of $\mathrm{OPHC} 2$ or $\mathrm{MPH}$, respectively. The activities of these mutants were measured using $\mathrm{MP}$ as substrate. Only three variants, $A b \mathrm{OPH}_{\mathrm{I} 211 \mathrm{~A}}, A b \mathrm{OPH}_{\mathrm{L} 156 \mathrm{M}}$ and $A b \mathrm{O}-$ $\mathrm{PH}_{\mathrm{H} 268 \mathrm{~L}}$, exhibited detectable OPH activities (Table 2), suggesting that residues 211,156 and 268 are crucial to $\mathrm{OPH}$ activity of $A b \mathrm{OPH}$. Mutation H268L of $A b \mathrm{OPH}$ just corresponds to the H250L of $\mathrm{PoOPH}$ that confirms the previously public conclusions (Luo et al. 2014). The key residues 211 and 156 that determine OPH activity were identified for the first time, especially the variant $A b \mathrm{OPH}_{\mathrm{I} 211 \mathrm{~A}}$ showed the most improved $\mathrm{OPH}$ activity (Table 2). Saturation mutagenesis at Ile211 and combination mutagenesis Ile211/L156M/H268L were performed subsequently to further improve OPH activity; however, all the other variants exhibited extremely lower $\mathrm{OPH}$ activities than $A b \mathrm{OPH}_{\mathrm{I} 211 \mathrm{~A}}$ (Additional file 1: Figure S5). 
Table 1 Data collection and refinement statistics

\begin{tabular}{|c|c|}
\hline & AbOPH \\
\hline \multicolumn{2}{|l|}{ Data collection statistics } \\
\hline Beamline/detector & Rigaku Micromax-007 HF\&Raxis IV++ \\
\hline Space group & $\mathrm{P} 2_{1} 2_{1} 2_{1}$ \\
\hline Unit cell ( $\AA$ /deg.) & $a=76.8, b=82.7, c=99.8, \alpha=\beta=\gamma=90.0$ \\
\hline Wavelength $(\AA)$ & 1.5418 \\
\hline Resolution range $(\AA)^{\mathrm{a}}$ & $50.00-2.00(2.05-2.00)$ \\
\hline Total/unique reflections & $43,098 / 2167$ \\
\hline Redundancy ${ }^{a}$ & $12.0(11.6)$ \\
\hline Completeness (\%) & $98.6(99.1)$ \\
\hline Mean $\mid / \sigma(\mid)^{\mathrm{a}}$ & $31.3(8.7)$ \\
\hline Wilson B factor $\left(\AA^{2}\right)$ & 25.2 \\
\hline Solvent content (\%) & 38.8 \\
\hline \multicolumn{2}{|l|}{ Refinement statistics } \\
\hline Resolution range $(\AA)^{\mathrm{a}}$ & $34.83-2.00(2.05-2.00)$ \\
\hline$R_{\text {work }}(\%)^{b}$ & 20.4 \\
\hline$R_{\text {free }}(\%)^{b}$ & 23.8 \\
\hline \multicolumn{2}{|l|}{ R.m.s. deviation } \\
\hline Bond lengths $(\AA)$ & 0.008 \\
\hline Bond angles (deg.) & 1.22 \\
\hline Average B factor $\left(\AA^{2}\right)$ & 26.0 \\
\hline \multicolumn{2}{|l|}{ Ramachandran plot } \\
\hline Favored (\%) & 96.5 \\
\hline Disallowed (\%) & 0.0 \\
\hline
\end{tabular}

a Numbers in parentheses are values for the highest-resolution shell

b $R_{\text {work }}=\sum|| F_{\mathrm{o}}-F_{\mathrm{c}}\left\|/ \mathrm{ac} F_{\mathrm{o}}\left|\cdot R_{\text {free }}=\sum_{\mathrm{T}}\right|\left|F_{\mathrm{o}}-F_{\mathrm{c}} \| / \mathrm{e}_{\mathrm{T}}\right| F_{\mathrm{o}} \mid\right.$, where $T$ is a test data set of $5 \%$ of the total reflections randomly chosen and set aside prior to refinement

The kinetic parameters of variants $A b \mathrm{OPH}_{\mathrm{I} 211 \mathrm{~A}}, A b \mathrm{O}$ $\mathrm{PH}_{\mathrm{L} 156 \mathrm{M}}$ and $A b \mathrm{OPH}_{\mathrm{H} 268 \mathrm{~L}}$ were determined by non-linear fitting. As shown in Table 2, the $K_{\mathrm{M}}$ values of these three mutants were similar, while the $k_{\text {cat }}$ of $A b \mathrm{OPH}_{\mathrm{I} 211 \mathrm{~A}}$ was significantly higher than those of $A b \mathrm{OPH}_{\mathrm{L} 156 \mathrm{M}}$ and $A b \mathrm{O}$ $\mathrm{PH}_{\mathrm{H} 268 \mathrm{~L}}$. The catalytic efficiency $\left(k_{\mathrm{cat}} / K_{\mathrm{M}}\right)$ of $A b \mathrm{OPH}_{\mathrm{I} 211 \mathrm{~A}}$ toward MP reached $0.9 \mathrm{~min}^{-1} \mu \mathrm{M}^{-1}$, which was nearly 50 times higher than those of $A b \mathrm{OPH}_{\mathrm{L} 156 \mathrm{M}}$ and $A b \mathrm{O}$ $\mathrm{PH}_{\mathrm{H} 268 \mathrm{~L}}$. The higher $k_{\text {cat }}$ of $A b \mathrm{OPH}_{\mathrm{I} 211 \mathrm{~A}}$ was considered as the main advantage for enzymatic hydrolysis of MP.

Activities of $A b \mathrm{OPH}_{\mathrm{I} 211 \mathrm{~A}}$ toward several OPs, lactones and esters were determined (Table 3; Additional file 1: Figure S6). Compared with wild-type $A b \mathrm{OPH}$, the $\mathrm{OPH}$ activity of $A b \mathrm{OPH}_{\mathrm{I} 211 \mathrm{~A}}$ was significantly enhanced and the lactonase activity was decreased, while the esterase activity was similar. $A b \mathrm{OPH}_{\mathrm{I} 211 \mathrm{~A}}$ showed significant activities toward MP and ethyl-paraoxon, while the activities of wild-type $A b \mathrm{OPH}$ toward those substrates were not detected. The activity of $A b \mathrm{OPH}_{\mathrm{I} 211 \mathrm{~A}}$ toward fenitrothion reached $0.54 \mu \mathrm{mol} \mathrm{min}^{-1} \mathrm{mg}^{-1}$, 11-fold higher than that of $A b \mathrm{OPH}$. Compared with $A b \mathrm{OPH}, A b \mathrm{OPH}_{\mathrm{I} 211 \mathrm{~A}}$ exhibited decreased lactonase activities toward 3,4-dihydrocoumarin, $\delta$-decanolactone and $\gamma$-nonanolactone, being 60, 93 and $89 \%$, respectively. Therefore, $A b \mathrm{O}$ $\mathrm{PH}_{\text {I211A }}$ exhibited a trade-off between its activities of lactonase and $\mathrm{OPH}$ in the evolutionary process.

\section{Docking studies}

In parallel with $A b \mathrm{OPH}_{\mathrm{I} 211 \mathrm{~A}}$, the alanine residue in position 211 was also found in many other enzymes with a high OPH activity (Luo et al. 2014; Dong et al. 2005). For example, a lactonase was actually engineered into an efficient $\mathrm{OPH}(\mathrm{PoOPH})$ without mutating the alanine residue to other residues (Luo et al. 2014). To investigate the possible mechanism of the improved OPH activity caused by I211A mutation, the MP molecule was docked into $A b \mathrm{O}$ $\mathrm{PH}_{\mathrm{I} 211 \mathrm{~A}}$ and $A b \mathrm{OPH}$, respectively, to simulate the protein-ligand interaction. In the $A b \mathrm{OPH}_{\mathrm{I} 211 \mathrm{~A}}-\mathrm{MP}$ complex (Fig. 3a), the phosphoryl sulfur atom of the substrate MP directly faces the catalytic $\beta$ zinc atom with a distance of $2.7 \AA$. The $p$-nitrophenyl moiety forms $\pi-\pi$ interactions with both benzene ring of Phe127 and the indole group of Tyr283, stabilizing the $p$-nitrophenyl in the leaving group pocket. The nitro group of MP is hydrogen bonded to Asn71 (2.6 $\AA$ ). One of the $O$-methyl substituents binds to the His157, implying a strong hydrogen bond between the O-methyl substituent and His157 (2.5 $)$. The conformation got from docking simulation implies that the substrate can form strong binding interactions with the catalytic zinc ion and the residues in the binding pocket, which is consistent with the previously catalytic assumptions (Luo et al. 2014; Gotthard et al. 2013).

Residue Ile 211 exhibits a distance of $9.7 \AA$ to the $\beta$ zinc atom and it is located in helix $\alpha 5$, just behind four additional residues 206-209. Based on structural alignment (Fig. $3 \mathrm{~b}$ ) of wild-type $A b \mathrm{OPH}$ and the mutant $A b \mathrm{OPH}_{\mathrm{I} 211 \mathrm{~A}}$, the carboxyl group of Ile211 in the $A b \mathrm{O}$ $\mathrm{PH}_{\mathrm{I} 211 \mathrm{~A}}-\mathrm{MP}$ complex structure represents a motion of $1.2 \AA$ compared with the $A b \mathrm{OPH}-\mathrm{MP}$ complex. The loop10 of $A b \mathrm{OPH}_{\mathrm{I} 211 \mathrm{~A}}$ moves closer to helix $\alpha 5(0.7 \AA)$, and the side chain of His157 located in loop10 moves toward the same direction $(1.4 \AA$ ) consequently. On the other hand, His157 of $A b \mathrm{OPHI}_{211 \mathrm{~A}}$ displays a distance of $2.5 \AA$ to the methoxyl group of MP, which is shorter than $3.1 \AA$ in the AbOPH-MP complex. Enlightened by the conformational alteration, a reasonable explanation for the increased OPH activity was proposed: I211A substitution decreases the size of the side chain, which offers enough space for the motion of the flexible loop10, and the conformational migration in loop10 makes the side chain of His157 move closer to the methoxyl group of MP, generating a much stronger interaction with MP. Therefore, the mutation I211A adjusted the local 

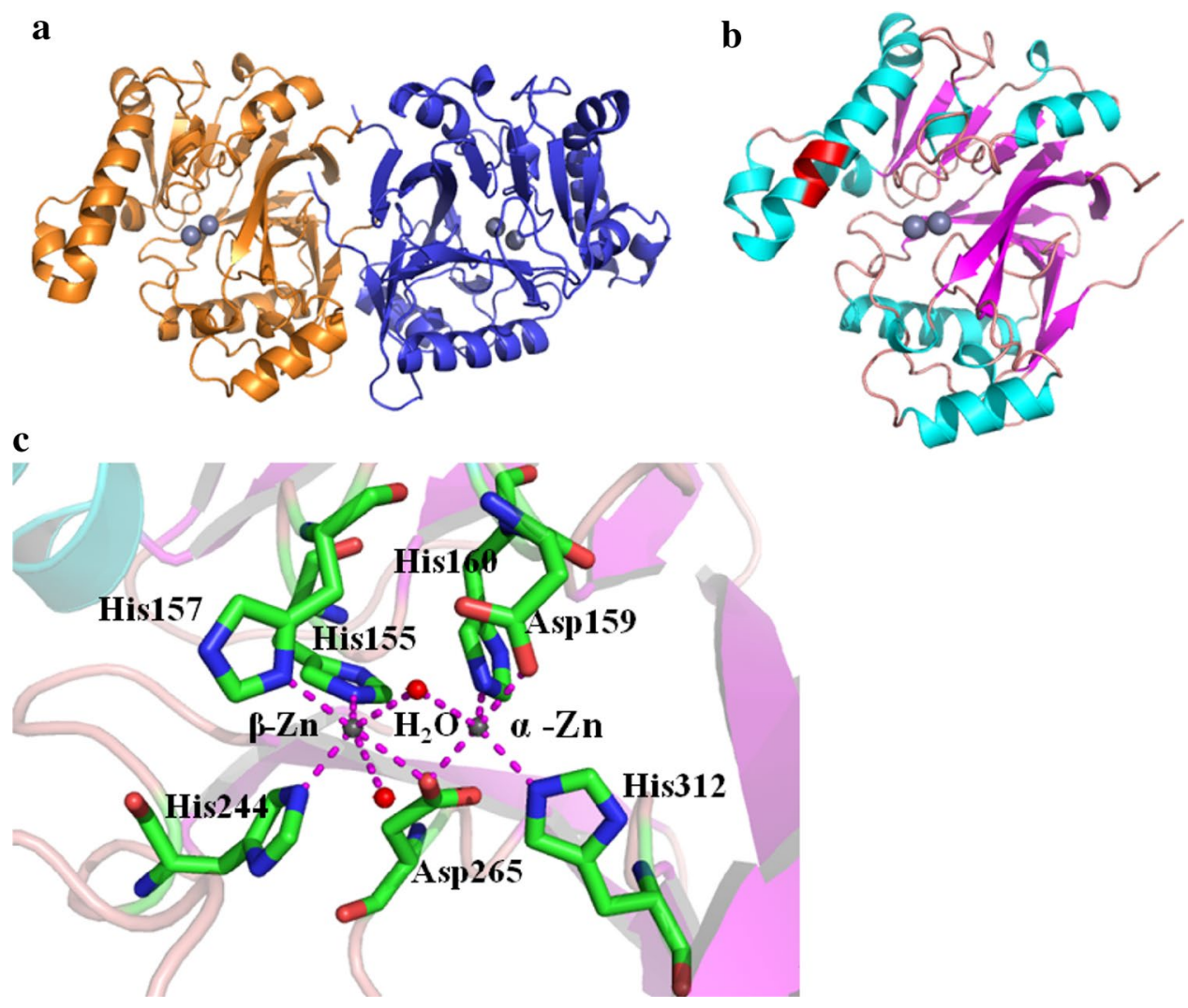

Fig. 2 Crystal structure of $A b O P H$. a General representation of the AbOPH dimer. The two metals of the active site are represented as gray spheres. $\mathbf{b}$ View of overall structure of the $\mathrm{AbOPH}$ monomer. $\alpha$ helices are colored in blue, $\beta$ sheets in magenta and loops in wheat color. The bimetallic center is shown as two gray spheres. Additional residues 206-209 are highlighted in red. c Active site of AbOPH. Residues are shown as sticks. The two metals are represented as gray balls and the two water molecules are shown as red spheres. The metal coordination sphere is shown as dashed line

Table 2 Specific activities and kinetic parameters of wild-type $A b O P H$ and its variants

\begin{tabular}{|c|c|c|c|c|}
\hline Enzymes & Specific activity (U/mg) & $K_{M}(\mu M)$ & $k_{\text {cat }}\left(\min ^{-1}\right)$ & $k_{\text {cat }} / K_{\mathrm{M}}\left(\min ^{-1} \mu \mathrm{M}^{-1}\right)$ \\
\hline $\mathrm{AbOPH}$ & ND & ND & $N D$ & - \\
\hline$A b O P H_{1211 \mathrm{~A}}$ & $1.060 \pm 0.023$ & $103 \pm 14$ & $93.2 \pm 3.2$ & 0.90 \\
\hline $\mathrm{AbOPH}_{\mathrm{L} 156 \mathrm{M}}$ & $0.0115 \pm 0.0010$ & $45.5 \pm 5.6$ & $0.862 \pm 0.031$ & 0.018 \\
\hline $\mathrm{AbOPH}_{\mathrm{H} 268 \mathrm{~L}}$ & $0.0104 \pm 0.0011$ & $56.7 \pm 7.0$ & $0.804 \pm 0.030$ & 0.013 \\
\hline
\end{tabular}

Specific activities of purified enzymes were measured toward $0.5 \mathrm{mM}$ MP

The kinetic parameters were determined by measuring purified enzyme activities toward MP at concentration from 0.01 to $1 \mathrm{mM}$ and fitting data to the MichaelisMenten equation. All enzymatic assays were performed at least in triplicate and average values were adopted

$N D$ no enzymatic activity was detected except spontaneous hydrolysis, in spite of the high enzyme loading $(2 \mathrm{mg} / \mathrm{mL})$

structure near the binding pocket and stabilizes the substrate binding with the specificity subsite of OPHs.

\section{Conclusions}

An "ancestral" enzyme, $A b \mathrm{OPH}$, was identified with high lactonase activity and faint $\mathrm{OPH}$ activity. Its crystal structure was resolved. Ile211 was identified as a key residue for acquiring new $\mathrm{OPH}$ activity through semi-rational redesign of $A b \mathrm{OPH}$ based on the crystal structure and alignment analysis. The $\mathrm{OPH}$ activity of single-mutated $A b \mathrm{OPH}_{\mathrm{I} 211 \mathrm{~A}}$ was remarkably enhanced, with a compromise of its native lactonase activity. This 
Table 3 Activities of $A b O P H$ and $A_{b O P H}{ }_{1211 A}$ toward various substrates

\begin{tabular}{|c|c|c|}
\hline Substrates & $A b O P H\left(\mu \mathrm{mol} \mathrm{min}^{-1} \mathrm{mg}^{-1}\right)$ & 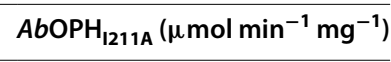 \\
\hline \multicolumn{3}{|l|}{ Phosphoesters } \\
\hline Methyl-parathion & ND & $1.68 \pm 0.08$ \\
\hline Ethyl-paraoxon & ND & $0.072 \pm 0.012$ \\
\hline Fenitrothion & $0.048 \pm 0.011$ & $0.543 \pm 0.045$ \\
\hline Malathion & ND & ND \\
\hline Dimethoate & ND & $N D$ \\
\hline Diazinon & ND & $N D$ \\
\hline \multicolumn{3}{|l|}{ Lactones } \\
\hline 3,4-Dihydrocoumarin & $996 \pm 22$ & $389 \pm 10$ \\
\hline$\delta$-Decanolactone & $54.1 \pm 6.1$ & $3.74 \pm 0.56$ \\
\hline$\gamma$-Nonanolactone & $14.8 \pm 2.0$ & $1.64 \pm 0.12$ \\
\hline \multicolumn{3}{|l|}{ Esters } \\
\hline para-Nitrophenyl butyrate & $3.73 \pm 0.11$ & $3.04 \pm 0.50$ \\
\hline 2-Naphthyl acetate & $3.52 \pm 0.46$ & $2.53 \pm 0.16$ \\
\hline
\end{tabular}

Data were obtained with purified proteins

$N D$ no enzymatic activity was detected except spontaneous hydrolysis regardless of the high enzyme loading (2 mg/mL)
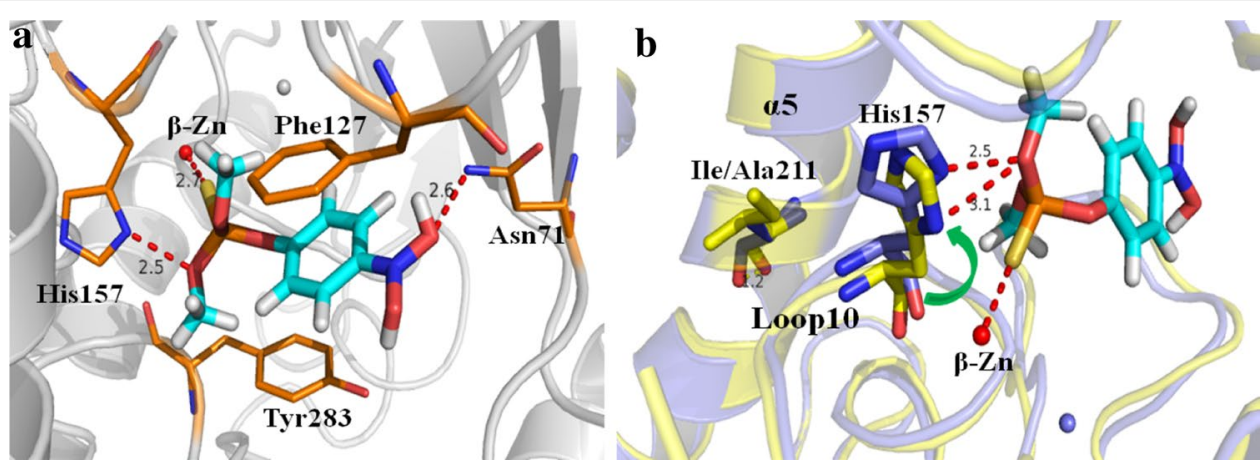

Fig. 3 Docking pose of MP in the structure of $A b O P H$ or $A b O P H_{1211 A}$. The substrate MP is shown as blue stick. a Details of the interaction of $A b O \mathrm{PH}_{1211 \mathrm{~A}}$ with MP in the active site. $\mathbf{b}$ Superposition of $\mathrm{AbOPH}-\mathrm{MP}$ (yellow) and $A b O P \mathrm{H}_{1211 \mathrm{~A}} \mathrm{MP}$ (blue). The green arrow represents the different conformation of side chain of His 157 in $\mathrm{AbOPH}-\mathrm{MP}$ and in the $\mathrm{AbOPH}_{1211 \mathrm{~A}}-\mathrm{MP}$ complex

result presents the emergence of new enzyme function by a simple mutation strategy and confirms the high possibility that OPH was evolved from its lactonase ancestor.

\section{Additional file}

Additional file 1. In the Supplemental Material Section results from the crystallization of the purified $\mathrm{AbOPH}$ protein and selection of the mutational amino acids as well as the activities comparison of $\mathrm{AbOPH}$ with its variants are presented.

\section{Abbreviations}

OPs: organophosphates; OPHs: organophosphorus hydrolases; $\mathrm{AbOPH}$ : a putative OPH from Acinetobacter sp.; OPHC2: OPH isolated from Pseudomonas pseudoalcaligenes; PoOPH: a putative OPH from Pseudomonas oleovorans; MP: methyl-parathion; MPHs: methyl-parathion hydrolases; PTEs: phosphotriesterases; PLLs: phosphotriesterase-like lactonases; PNPB: para-nitrophenyl butyrate.

\section{Authors' contributions}

$J C$ drafted the manuscript and made substantial contributions to acquisition, analysis and interpretation of data; X-JL and JP designed the study and were responsible for the revision of the manuscript; QC conducted the experiments; $\mathrm{J}-\mathrm{HX}$ and JZ provided experimental guidance. All authors read and approved the final manuscript.

\section{Author details}

${ }^{1}$ State Key Laboratory of Bioreactor Engineering, Shanghai Collaborative Innovation Center for Biomanufacturing, East China University of Science and Technology, Shanghai 200237, China. ${ }^{2}$ Shanghai Institute of Organic Chemistry, Chinese Academy of Sciences, Shanghai 200032, China.

\section{Acknowledgements}

This work was financially supported by the Ministry of Science and Technology, P.R. China (Nos. 2012AA022206C and 2011CB710800) and The Open Project from the State Key Laboratory of Bioorganic and Natural Product Chemistry, Shanghai 200032, China. 


\section{Compliance with ethical guidelines}

\section{Competing interests}

The authors declare that they have no competing interests.

Received: 22 June 2015 Accepted: 25 August 2015

Published online: 15 September 2015

\section{References}

Afriat L, Roodveldt C, Manco G, Tawfik DS (2006) The latent promiscuity of newly identified microbial lactonases is linked to a recently diverged phosphotriesterase. Biochemistry 45:13677-13686

Bar-Rogovsky H, Hugenmatter A, Tawfik DS (2013) The evolutionary origins of detoxifying enzymes: the mammalian serum paraoxonases (PONs) relate to bacterial homoserine lactonases. J Biol Chem 288:23914-23927

Ben-David M, Elias M, Filippi JJ, Dunach E, Silman I, Sussman JL, Tawfik DS (2012) Catalytic versatility and backups in enzyme active sites: the case of serum paraoxonase 1. J Mol Biol 418:181-196

Cheng TC, DeFrank JJ, Rastogi VK (1999) Alteromonas prolidase for organophosphorus G-agent decontamination. Chem Biol Interact 119:455-462

Dong YJ, Bartlam M, Sun L, Zhou YF, Zhang ZP, Zhang GG, Rao Z, Zhang XE (2005) Crystal structure of methyl parathion hydrolase from Pseudomonas sp. WBC-3. J Mol Biol 353:655-663

Du FL, Yu HL, Xu JH, Li CX (2014) Enhanced limonene production by optimizing the expression of limonene biosynthesis and MEP pathway genes in E. coli. Bioresour Bioprocess 1:10

Dumas DP, Caldwell SR, Wild JR, Raushel FM (1989) Purification and properties of the phosphotriesterase from Pseudomonas diminuta. J Biol Chem 264:19659-19665

Elias M, Dupuy J, Merone L, Mandrich L, Porzio E, Moniot S, Rochu D, Lecomte C, Rossi M, Masson P, Manco G, Chabriere E (2008) Structural basis for natural lactonase and promiscuous phosphotriesterase activities. J Mol Biol 379:1017-1028

Emsley P, Cowtan K (2004) Coot: model-building tools for molecular graphics. Acta Crystallogr. D 60:2126-2132

Gotthard G, Hiblot J, Gonzalez D, Elias M, Chabriere E (2013) Structural and enzymatic characterization of the phosphotriesterase OPHC2 from pseudomonas pseudoalcaligenes. PLoS ONE 8:e77995

Hawwa R, Larsen SD, Ratia K, Mesecar AD (2009) Structure-based and random mutagenesis approaches increase the organophosphate-degrading activity of a phosphotriesterase homologue from Deinococcus radiodurans. J Mol Biol 393:36-57

Hong L, Zhang JJ, Zhang SJ, Zhang XE, Zhou NY (2005) Plasmid-borne catabolism of methyl parathion and $p$-nitrophenolin Pseudomonas sp. strain WBC-3. Biochem Biophys Res Commun 334:1107-1114
Khersonsky O, Tawfik DS (2005) Structure-reactivity studies of serum paraoxonase PON1 suggest that its native activity is lactonase. Biochemistry 44:6371-6382

Luo XJ, Kong XD, Zhao J, Zhou JH, Xu JH (2014) Switching a newly discovered lactonase into an efficient and thermostable phosphotriesterase by simple double mutations His250lle/lle263Trp. Biotechnol Bioeng 111:1920-1930

McCoy AJ, Grosse-Kunstleve RW, Adams PD, Winn MD, Storoni LC, Read RJ (2007) Phaser crystallographic software. J Appl Crystallogr 40:658-674

Merone L, Mandrich L, Porzio E, Rossi M, Muller S, Reiter G, Worek F, Manco G (2010) Improving the promiscuous nerve agent hydrolase activity of a thermostable archaeal lactonase. Bioresour Technol 101:9204-9212

Murshudov GN, Vagin AA, Dodson EJ (1997) Refinement of macromolecular structures by the maximum-likelihood method. Acta Crystallogr D 53:240-255

Omburo GA, Kuo JM, Mullins LS, Raushel FM (1992) Characterization of the zinc binding site of bacterial phosphotriesterase. J Biol Chem 267:13278-13283

Otwinowski Z, Minor W (1997) Processing of X-ray diffraction data collected in oscillation mode. Methods Enzymol 276:307-326

Raushel FM (2002) Bacterial detoxification of organophosphate nerve agents. Curr Opin Microbiol 5:288-295

Roodveldt C, Tawfik DS (2005) Shared promiscuous activities and evolutionary features in various members of the amidohydrolase superfamily. Biochemistry 44:12728-12736

Sapozhnikova Y, Bawardi O, Schlenk D (2004) Pesticides and PCBs insediments and fish from the Salton Sea, California, USA. Chemosphere 55:797-809

Sapozhnikova Y, Zubcov N, Hungerford S, Roy LA, Boicencob N, Zubcovb E, Schlenka D (2005) Evaluation of pesticides and metals in fish of the Dniester River, Moldova. Chemosphere 60:196-205

Singh BK (2008) Organophosphorus-degrading bacteria: ecology and industrial applications. Nat Rev Microbiol 7:156-164

Singh BK, Walker A (2006) Microbial degradation of organophosphorus compounds. FEMS Microbiol Rev 30:428-471

Vyas NK, Nickitenko A, Rastogi VK, Shah SS, Quiocho FA (2010) Structural insights into the dual activities of the nerve agent degrading organophosphate anhydrolase/prolidase. Biochemistry 49:547-559

Zhang Y, An J, Ye W, Yang G, Qian ZG, Chen HF, Cui L, Feng Y (2012) Enhancing the promiscuous phosphotriesterase activity of a thermostable lactonase (GkaP) for the efficient degradation of organophosphate pesticides. Appl Environ Microbiol 78:6647-6655

\section{Submit your manuscript to a SpringerOpen ${ }^{\odot}$ journal and benefit from:}

- Convenient online submission

- Rigorous peer review

- Immediate publication on acceptance

- Open access: articles freely available online

- High visibility within the field

- Retaining the copyright to your article

Submit your next manuscript at $>$ springeropen.com 